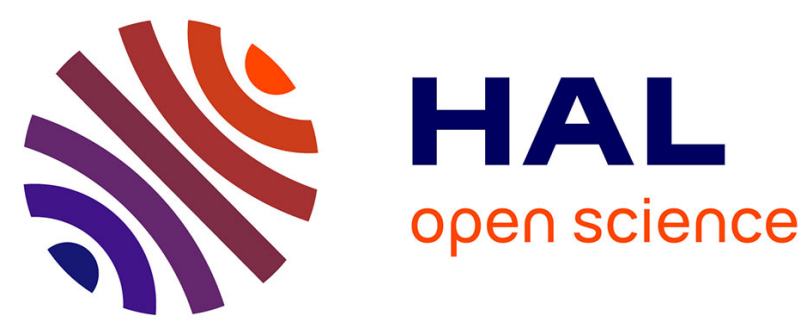

\title{
Pragmatics, Mental Models and One Paradox of the Material Conditional
}

\author{
Jean-François Bonnefon, Guy Politzer
}

\section{To cite this version:}

Jean-François Bonnefon, Guy Politzer. Pragmatics, Mental Models and One Paradox of the Material Conditional. Mind and Language, 2011, 26 (2), pp.141-155. ijn_01295435

\section{HAL Id: ijn_01295435 \\ https://hal.science/ijn_01295435}

Submitted on 1 Apr 2016

HAL is a multi-disciplinary open access archive for the deposit and dissemination of scientific research documents, whether they are published or not. The documents may come from teaching and research institutions in France or abroad, or from public or private research centers.
L'archive ouverte pluridisciplinaire HAL, est destinée au dépôt et à la diffusion de documents scientifiques de niveau recherche, publiés ou non, émanant des établissements d'enseignement et de recherche français ou étrangers, des laboratoires publics ou privés. 


\title{
Pragmatics, Mental Models, and one Paradox of the Material Conditional
}

\author{
Jean-François Bonnefon ${ }^{1}$ (CNRS, Université de Toulouse) \\ Guy Politzer (CNRS, Institut Jean Nicod)
}

Word count: 6,178

\footnotetext{
${ }^{1}$ To whom correspondence should be addressed: Cognition, Langues, Langage et Ergonomie, Maison de la recherche, 5 allées A. Machado, 31058 Toulouse Cedex 9, France. Phone +33 561503601 E-mail: bonnefon@univ-tlse2.fr. We thank Paul Egré for his thoughtful comments, two anonymous reviewers for their useful suggestions, and Phil Johnson-Laird for his thorough and generous reading of our manuscript.
} 


\begin{abstract}
Most instantiations of the inference ' $y$; so if $x, y$ ' seem intuitively odd, a phenomenon known as one of the paradoxes of the material conditional. A common explanation of the oddity, endorsed by Mental Model theory, is based on the intuition that the conclusion of the inference throws away semantic information. We build on this explanation to identify two joint conditions under which the inference becomes acceptable: (a) the truth of $x$ has bearings on the relevance of asserting $y$; and (b) the speaker can reasonably be expected not to be in a position to assume that $x$ is false. We show that this dual pragmatic criterion makes accurate predictions, and contrast it with the criterion defined by the mental model theory of conditionals, which we show to be inadequate.
\end{abstract}




\section{Introduction}

One well-known 'paradox' of the material conditional consists of accepting that 'if $x, y$ ' follows from $y$ by a valid rule of inference. This inference is deemed paradoxical because most of its instantiations in natural language are intuitively odd. For example:

(1) ?Today is Monday; so, if it is snowing outside, today is Monday. ${ }^{1}$

Importantly, though, other instantiations of the inference appear intuitively acceptable, for example:

(2) Viv did not play soccer; so, if she played a game, Viv did not play soccer.

This fact is given prominent importance in one major psycholinguistic theory of conditionals, mental model theory (Johnson-Laird \& Byrne, 2002). One critical argument commonly advanced in favor of the mental model theory of conditionals is its ability to predict which instantiations of the paradoxical inference seem

\footnotetext{
${ }^{1}$ All through this article, we will use the formulation 'if $x, y$ ' for conditionals, and the formulation ' $y$; so if $x$, $y$ ' for the paradoxical inference. Natural language offers a number of other possible formulations of the conditional. Some of these formulations strengthen or weaken the paradox (e.g., 'if $x$ then $y$ ' and 'even if $x, y$ ', respectively) to the point where it becomes impractical to generate acceptable or unacceptable examples, respectively. We settle on the formulation that apparently offers the greatest flexibility for the purpose of generating acceptable and unacceptable examples. We content ourselves with noting that the linguistic formulation of the conditional is a factor in the acceptability of the paradoxical inference, whose interaction with our variables of interest is left for future research.
} 
acceptable, and why. The mental model account of the paradox remains controversial, though (Johnson-Laird, Byrne, \& Girotto, 2009; Politzer, 2007, 2009), and is still waiting for a systematic assessment. In this article, we contrast the mental model account of the paradox to our own pragmatic account. We show that the mental model criterion is neither necessary nor sufficient to predict the acceptability of the paradoxical inference, which is adequately predicted by our dual pragmatic criterion. ${ }^{2}$ While we focus on mental model theory in the course of the article, we will also discuss the contribution of our analysis to other psychological theories of natural language conditionals, which have more drastically distanced themselves from interpreting the conditional as a material implication.

In Section 2, we introduce the mental model account and its criterion for the acceptability of the paradoxical inference, which we express as a binary condition that we call condition $m$. In Section 3, we introduce the fundamental hypothesis underlying our pragmatic account. In a nutshell, we argue that a conditional sen-

\footnotetext{
${ }^{2}$ The paradox that we address in this article is usually presented alongside with a second paradox, that of inferring 'if $x, y$ ' from ' $\neg x$ '. We will not address this second paradox in the present article. We believe that the acceptability of these paradoxical inferences is determined by pragmatic, conversational principles; and that the conversational principles that govern the acceptability of the first inference do not necessarily govern the acceptability of the second. Since we do not believe in a single, unified theory of the acceptability of both paradoxical inferences, we do not feel compelled to offer one. Mental model theory does offer a unified account of the two paradoxes, but this unified account is necessarily incorrect if, as we intend to show, it fails on at least one of the paradoxes. Finally, we note that although it offers a unified theory of both paradoxes, mental model theory restricts itself to the first paradox when it comes to offering examples of acceptable instantiations.
} 
tence 'if $x, y$ ' uttered in the context of $y$, or asserted as following from $y$, seems odd when the speaker is asserting that $y$ is unconditionally true, and then the truth of $x$ has bearings on the truth of $y$. The paradox should disappear, then, when the conditional does not express that the truth of $x$ has bearings on the truth of $y$, but rather that the truth of $x$ has bearings on the relevance of asserting $y$ (where relevance can derive from epistemic utility or practical utility).

This hypothesis leads us to predict the dissolution of the paradox from a dual criterion that we express under the form of two binary conditions $p_{1}$ and $p_{2}$. In Section 4, we examine the eight possible cases that correspond to the satisfaction (or non-satisfaction) of the binary conditions $m, p_{1}$, and $p_{2}$. We show that the paradox disappears only when $p_{1}$ and $p_{2}$ are both satisfied (including the case where $m$ is not satisfied), and remains in all other cases (including the cases where $m$ is satisfied).

Before we proceed, we wish to exclude one specific case from our analyses, that of when $y$ is known to be false. It is correct that the inference from $\perp$ to if $x$ then $\perp$ ' (where $\perp$ denotes the contradiction) is both logically valid and intuitively odd, and could thus be considered as a regular case of the paradox. The logical validity of this inference, though, is unrelated to the interpretation of the conditional, and is rather due to the ex falso quodlibet principle of classical logic. Anything follows validly from $\perp$, including indeed 'if $x$ then $\perp$.' In this article, we are interested in a paradox that arises from the interpretation of the conditional, and not in the paradoxes that arise from the ex falso quodlibet principle; and we will accordingly discard that case from our subsequent analyses. 


\section{The Mental Model Account}

The mental model theory of conditionals (Johnson-Laird \& Byrne, 1991, 2002) assumes that the paradoxical inference is counterintuitive because it throws away semantic information: That is, the conditional 'If $x, y$ ' introduces a possibility that was ruled out by the original premise $y$. Consider for example the premise 'Today is Monday.' From this premise, it would seem anomalous to infer that 'If we have pasta for lunch today is Monday':

(3) ?Today is Monday; so, if we have pasta for lunch, today is Monday.

According to the mental model theory, the conditional 'If we have pasta for lunch then today is Monday' refers to three possibilities:

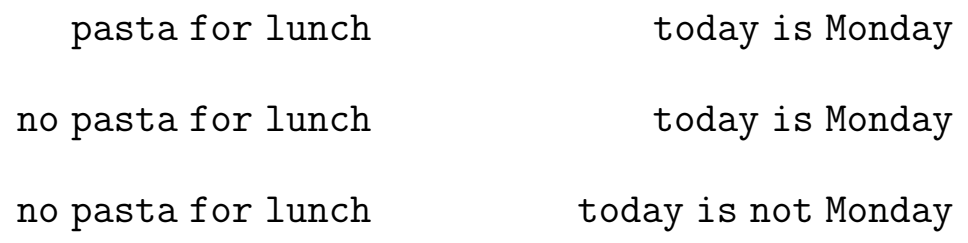

The reason why (3) is counterintuitive, according to the mental model account, is because the third possibility above, < no pasta for lunch, today is not Monday $>$ was ruled out by the original premise 'Today is Monday.' More generally, the inference ' $y$; so if $x, y$ ' is intuitively anomalous because $<\neg x, \neg y>$ is a model of the conditional, while $\neg y$ was ruled out by the original premise $y$. Inferring the conditional from this premise would amount to throwing away semantic informa- 
tion. $^{3}$

The mental model account then makes a clear prediction about when individuals will readily endorse the paradox. If common knowledge rules out $\langle\neg x, \neg y\rangle$ as a model of 'If $x, y$ ', then the inference ' $y$; so if $x, y$ ' should seem acceptable, because it no longer throws away semantic information. Let us define this as 'condition $m$ ':

Definition 1 (Condition $m$ ). The inference ' $y$; so if $x$, $y$ ' is intuitively acceptable if and only if $(m)$ the possibility $<\neg x, \neg y>i$ s ruled out by common knowledge and thus cannot be a model of the conditional 'If $x, y$ '.

The definition above indicates that $m$ is a necessary and sufficient condition for the intuitive acceptability of the inference. This characterisation is consistent with the most recent exposition of the mental model account (Johnson-Laird et al., 2009), which states both the sufficient and the necessary parts of the condition:

[Sufficiency] In what cases, should individuals accept a 'paradox' immediately $[\ldots]$ ? The model theory answers with a clear prediction: when the conclusion does not add a disjunctive alternative in which the premise fails to hold. (p. 78)

[Necessity] The crux is simple: if the conclusion of a valid inference throws information away by adding a disjunctive alternative to the possibilities con-

\footnotetext{
${ }^{3}$ Mental model theory offers the same explanation of why the inference from $x$ to the disjunction $x$ or $y$ can feel intuitively anomalous: One model of the disjunction is $\langle\neg x, y\rangle$, which was ruled out by the original premise $x$.
} 
sistent with the premises, then it should seem odd, and individuals should balk at it. The challenge to critics is to find a counterexample. (p. 78$)^{4}$

Let us now illustrate the workings of condition $m$ with Conditional (4):

(4) If Viv played a game, Viv did not play soccer.

The model $<\neg$ game, soccer $>$ cannot be part of the representation of (4), because it is semantically inconsistent: one cannot play soccer without playing a game. Therefore, the models of (4) are:

$\begin{array}{ll}\text { game } & \neg \text { soccer } \\ \neg \text { game } & \neg \text { soccer }\end{array}$

Because none of these models contains soccer, the inference (5) should not seem anomalous:

(5) Viv did not play soccer; so, if Viv played a game, Viv did not play soccer.

And indeed, (5) does not sound as odd as (3). The fact that the mental model account could generate an acceptable instance of the paradox is an impressive

\footnotetext{
${ }^{4}$ Although this last quote unambiguously expresses the necessity of condition $m$, we ackowledge the possibility that it might have gone beyond the intention of the authors, who did not construe $m$ as a necessary condition for the acceptability of the inference. If this is the case, then the mental model account does not make any prediction as to whether the paradox appears when condition $m$ is violated, and thus does not make any prediction in four of the eight cases that we will review in the rest of this article. Our own account still does, of course.
} 
feat, which speaks powerfully for the account. In the rest of this article, though, we are going to claim that the mental model account of the paradox is incorrect. We will show that condition $m$ is neither necessary nor sufficient to make ' $y$; so if $x, y^{\prime}$ an intuitively acceptable inference; and we will offer as a replacement a pragmatic account which will predict when and why the paradox disappears.

\section{The Pragmatic Account}

The fundamental idea behind the mental model account is that it is a waste of information to derive the less informative conclusion 'If $x, y$ ' from the more informative premise $y$. This idea concurs with our basic hypothesis presented in the introduction. Pragmatically speaking, the inclusion of the conditional in (6) is bound to be a waste of the listener's time and attention:

(6) ?I had bacon this morning; so, if I had juice, I had bacon this morning.

In the language of relevance theory (Sperber \& Wilson, 1986/1995), the second part of the statement requires additional processing effort that does not deliver additional cognitive effects. ${ }^{5}$ Generally speaking, when $y$ is already asserted as unconditionally true, no additional information is communicated by asserting that

\footnotetext{
${ }^{5}$ Our intention is not to introduce neo-Gricean pragmatics as a general alternative to mental model theory. We are agnostic about the general level of consistency between mental model theory and neo-gricean pragmatics. Nevertheless, the account of the paradox that we offer in this article does derive from Gricean and neo-Gricean pragmatics, and is in this specific case pitted against the mental model account.
} 
the truth of $x$ has bearings on the truth of $y$.

It is not always the case, though, that a statement of the form 'If $x, y$ ' expresses that the truth of $x$ has bearings on the truth of $y$. Some conditionals express instead that the truth of $x$ has bearings on the relevance of the assertion that $y$. This is typically the case in 'biscuit' conditionals (Austin, 1970; DeRose \& Grandy, 1999; Siegel, 2006):

(7) a. There are biscuits on the sideboard if you want them;

b. If you are thirsty now, there are beers in the fridge;

c. If you need help, my name is Bob.

Clearly, Bob does not mean by (7-c) that the listener's need for help has bearings on whether his own name is Bob. What Bob means is rather that whether the listener needs help has bearings on the relevance of asserting that his own name is Bob. Similarly, the speaker who is uttering (7-b) means that whether the listener is thirsty has bearings on whether it would be relevant to assert that there are beers in the fridge. Note now that the felicity of these interpretations requires that the speaker is not in a position to assume that the antecedent of the conditional is false. This condition accounts for the difference between (8-a) and (8-b):
a. If you are thirsty now, there are beers in the fridge;
b. ?If I am thirsty now, there are beers in the fridge.

We have claimed so far that a conditional 'If $x$, then $y$ ' does not always express that the truth of $x$ has bearings on the truth of $y$, as some conditionals express 
instead that the truth of $x$ has bearings on the relevance of asserting $y$. We now note that the relevance of asserting $y$ can derive either from the basic epistemic utility of learning that $y$, or additionally from the practical utility of knowing that $y$.

In example (7-a-c), the relevance of asserting $y$ derives from the practical utility this information has for the listener. Indicating the whereabouts of the beers, for example, would have practical utility for a listener whose goal is to quench her thirst. Accordingly, (7-b) expresses that the listener's thirst has bearings on the practical relevance of asserting that there are beers in the fridge. In other cases, the relevance of asserting $y$ derives from the mere epistemic utility of learning that $y$. This happens when there is no immediately manifest goal of the listener that would be served by knowing that $y$, bar the generic goal of augmenting one's knowledge about the world. Consider for example:

(9) If you've not seen Amy yet, she's dyed her hair red.

Conditional (9) does not, of course, express that whether the listener has seen Amy has bearings on the fact that Amy dyed her hair red. It rather expresses that whether the listener has seen Amy already, has bearings on the relevance of asserting that Amy dyed her hair red. Indicating that Amy has dyed her hair red would have epistemic utility for a listener who would not already be in a possession of this piece of knowledge, typically because this listener would not have seen Amy already. ${ }^{6}$

\footnotetext{
${ }^{6}$ One could imagine contexts wherein knowing that Amy dyed her hair red would have practical utility for the listener in addition to epistemic utility, and such contexts would make other
} 
Once again, the felicity of this interpretation would be compromised if the speaker was in a position to assume that the antecedent of the conditional is false, which accounts for the difference between (10-a) and (10-b):
a. If you've not seen Amy yet, she's dyed her hair red;
b. ?If we've not seen Amy yet, she's dyed her hair red.

We are now in a position to get back to the paradox. We have remarked that the statement ' $y$; so if $x, y$ ' is pragmatically incongruous when it expresses that the truth of $x$ has bearings on the truth of $y$. Exploiting the remarkable case of biscuit conditionals, we have pointed out, however, that the conditional 'If $x, y$ ' can have pragmatic import over and above the assertion that $y$, when it expresses that the truth of $x$ has bearings on the relevance of asserting $y$. In line with Gricean and neo-Gricean pragmatics, we have shown further that this pragmatic import can be obtained when the relevance of asserting $y$ derives from its epistemic utility for the listener, or from its practical utility for the listener; and that it requires that the speaker can plausibly ignore whether $x$ is false. We suggest that the statement ' $y$; so if $x, y$ ' is pragmatically acceptable when these conditions are met, and we thus suggest replacing condition $m$ with conditions $p_{1}$ and $p_{2}$ :

Definition 2 (Conditions $p_{1}$ and $p_{2}$ ). The inference ' $y$; so if $x$, $y$ ' is intuitively acceptable if and only if $\left(p_{1}\right)$ the truth of $x$ has bearings on the relevance of asserting $y$; and $\left(p_{2}\right)$ the speaker can reasonably be expected not to be in a position antecedents appropriate. For example, 'If you placed a bet on Amy's new hair colour, she's dyed her hair red.' 
to assume that $x$ is false.

\section{Teasing Out the Two Accounts}

In order to tease out the mental model account and the pragmatic account, we must review the eight cases defined by the satisfaction or violation of conditions $m, p_{1}$, and $p_{2}$. As displayed in Table 1, there are four cases wherein the two accounts make similar predictions, two critical cases wherein they make different predictions, and two special cases that cannot be constructed due to the logical relation between condition $m$ and condition $p_{1}$, which we will address later on.

\subsection{Cases of Agreement}

When all three conditions $m, p_{1}$, and $p_{2}$ are met, both accounts naturally predict that the statement ' $y$; so if $x, y$ ' sounds acceptable. This is Case 8 of Table 1 . The classic example offered by the mental model account falls in this category:

(11) Viv did not play soccer; so, if she played a game, Viv did not play soccer.

Condition $m$ is met because the possibility $<\neg x, \neg y>$, here $<\neg$ game, soccer $>$, is ruled out by common knowledge about the semantics of 'game' and 'soccer.'

Condition $p_{1}$ requires that the truth of $x$ has bearings on the epistemic or practical relevance of asserting $y$. It is clearly the case here that if $x$ were known to be false (i.e., if Viv was known not to have played any game), the assertion of 


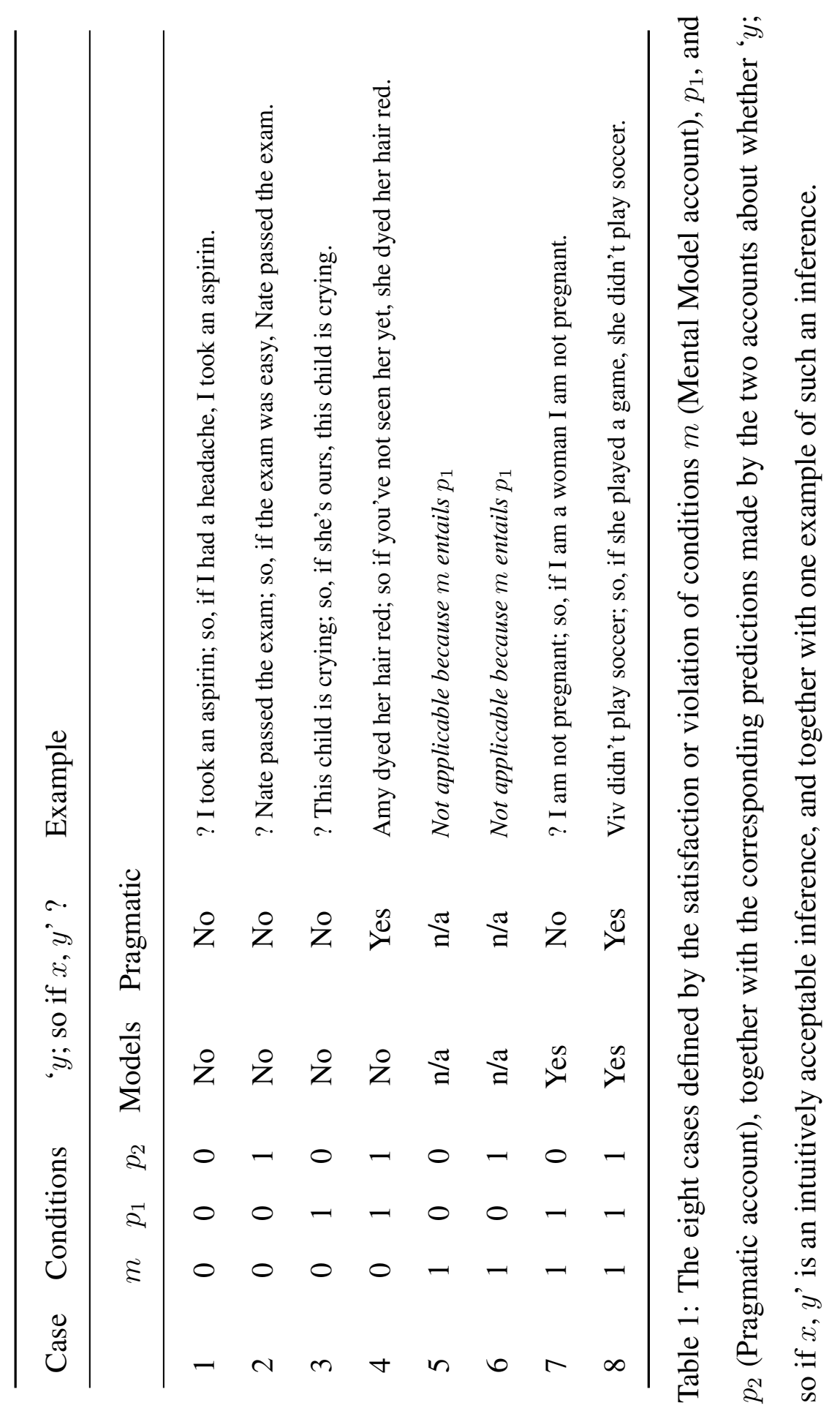


$y$ (she did not play soccer) would have no epistemic relevance. ${ }^{7}$ Example (11) thus meets condition $p_{1}$ of the pragmatic account, one quick test of which is to tentatively add 'at all' to the antecedent of the conditional, as in (12):

(12) Viv did not play soccer; so, if she played a game at all, Viv did not play soccer.

Finally, (12) gives no indication that the speaker might be in a position to assume that Viv did not play a game, thus satisfying condition $p_{2}$.

So far, we have only addressed one case of agreement between the two accounts, wherein they both predict the paradox to vanish. Table 1 also displays three cases wherein both accounts predict that ' $y$; so if $x, y$ ' is intuitively incongruous. Case 1 denotes situations that violate all three conditions $m, p_{1}$, and $p_{2}$, as in (13):

(13) ?I took an aspirin; so, if I had a headache, I took an aspirin.

Condition $m$ is violated because the possibility $<\neg$ headache, $\neg$ aspirin $>$ is entirely consistent with common knowledge. Condition $p_{1}$ is violated because the informational or practical value of asserting 'I took an aspirin' does not depend

\footnotetext{
${ }^{7}$ The fact that $y$ contains a negation is not alien to the appeal of example (11). Because they cannot spend their time denying everything they know to be false, people are especially sensitive to the pragmatic presuppositions required for negations to make conversational sense. For conditional reasoning experiments capitalising on these pragmatic presuppositions, see Bonnefon and Villejoubert (2007).
} 
in any immediately salient way on the fact that $\mathrm{I}$ had a headache. Condition $p_{2}$ is violated because I, the speaker, am in a position to know for a fact whether I did not have a headache prior to taking the aspirin.

Case 2 denotes situations wherein condition $m$ and $p_{1}$ are violated, although condition $p_{2}$ is satisfied, as in (14), where nothing indicates that the speaker might assume that the exam was in fact hard:

(14) ?Nate passed the exam; so, if the exam was easy, Nate passed the exam.

Finally, Case 3 denotes situations where condition $m$ and $p_{2}$ are violated, although condition $p_{1}$ is satisfied, as in (15):

(15) ?This child is crying; so, if she's ours, this child is crying.

Condition $m$ is violated because it is clearly possible that a child who is not that of the speaker and the listener is not crying. Condition $p_{1}$ is met because whether the child is that of the listener clearly has bearings on the practical relevance of asserting that the child is crying: The listener plausibly has the general goal of monitoring the well-being of her child, but not as plausibly that of monitoring the well-being of other parents' children. Finally, condition $p_{2}$ is violated because the speaker should know whether the crying child is his. Note now the difference between (15) and (16):

(16) This child is crying; so, if she's yours, this child is crying. 
Example (16) does not sound as odd as example (15), for reasons that the pragmatic account can explain, but that elude the mental model acccount. It reflects one of the critical cases that we will scrutinize in the next section.

\subsection{Critical Cases}

There are two critical cases wherein the mental model account and the pragmatic account make opposite predictions. In case 4, both conditions $p_{1}$ and $p_{2}$ are met, but condition $m$ is violated. Accordingly, the model account predicts that ${ }^{6} y$; so if $x$, $y$ ' sounds unacceptable, whereas the pragmatic account predicts that ' $y$; so if $x, y$ ' sounds acceptable. Conversely, in case 7 , condition $m$ is met, and so is condition $p_{1}$, but condition $p_{2}$ is violated. Accordingly, the pragmatic account predicts that ' $y$, so if $x$ then $y$ ' is incongruous, whereas the model account predicts that it is acceptable.

We first consider case 4 , of which (17-a-c) are three examples:
a. Amy dyed her hair red; so, if you've not seen her yet, Amy dyed her hair red;
b. This child is crying; so, if she's yours, this child is crying.
c. There is beer in the fridge; so, if you want some, there is beer in the fridge.

In all these examples, the model $\langle\neg x, \neg y>$ is perfectly consistent with world knowledge, and is thus part of the representation of the conditional 'if $x, y$ '. Indeed, there is nothing intrinsically implausible, let alone impossible, with the situ- 
ations captured by the model $\langle\neg x, \neg y>$ : Amy, whose hair is not dyed red, being seen by someone; a child who is not crying, and who is not that of a listener; a fridge that is devoid of beers, at a moment someone has no desire for beers.

Thus, the possibility $<\neg x, \neg y>$ has to be part of the representation of the conditionals featured in (17-a-c), and the conditional 'If $x, y$ ' is bound to throw away semantic information as compared to the premise ' $y$ '' As a consequence, the mental model account predicts that all three examples (17-a-c) should sound incongruous and unacceptable. Clearly, this prediction is not supported by our intuitive reaction to these examples.

The pragmatic account, in contrast, predicts examples (17-a-c) to be acceptable, because they all meet conditions $p_{1}$ and $p_{2}$. In (17-a), whether the listener has seen Amy already, has bearings on the epistemic relevance of asserting that Amy dyed her hair red. In (17-b), whether the child is that of the listener has bearings on the practical relevance of asserting that the child is crying. Likewise, in (17-c), whether the listener is thirsty has bearings on the practical relevance of asserting that there is beer in the fridge. Thus, condition $p_{1}$ is satisfied in all three examples.

In parallel, condition $p_{2}$ is also satisfied in the three examples. Nothing in example (17-a) indicates that the speaker is in a position to know or assume that the listener did not see Amy already; and, mutatis mutandis, the same can be said about examples (17-b-c). Because conditions $p_{1}$ and $p_{2}$ are met for all three examples (17-a-c), the pragmatic account (correctly) predicts that they should be intuitively acceptable.

We now turn to case 7 in Table 1 , wherein condition $m$ is met, as well as 
condition $p_{1}$, but not condition $p_{2}$. In such a situation, the model account predicts that we should not flinch at the statement ' $y$; so if $x, y$,' whereas the pragmatic account predicts the opposite. Now consider (18):

?I am not pregnant; so, if I am a woman I am not pregnant.

Condition $m$ is unambiguously met by example (18). Indeed, the model $<$ $\neg$ woman, pregnant $>$ is ruled out by common knowledge, and is not part of the representation of 'If I am a woman I am not pregnant.' Thus, the conditional does not throw away information as compared to the premise 'I am not pregnant,' and (18) should not sound incongruous according to the model account. The oddness of (18), though, is beyond reasonable doubt. The pragmatic account predicts this oddness, based on the violation of condition $p_{2}$, i.e., based on the common expectation that speakers are in a position to know for a fact about their gender. In contrast, $p_{2}$ is satisfied if one changes (18) minimally into (19):

This person is not pregnant; so, if this person is a woman, she is not pregnant.

Accordingly, and as predicted, (19) is not incongruous. Note that condition $p_{1}$ of the pragmatic account is met by examples (18) and (19), because the statement 'I am not pregnant' would have no epistemic relevance if the speaker was not a woman; and mutatis mutandis for (19). As we have hinted on several occasions, the satisfaction of $p_{1}$ is in fact entailed by the satisfaction of $m$. We turn to that issue in the next section, where we consider the last two, special cases in Table 1, 
which cannot be constructed due to the logical relation between $m$ and $p_{1}$.

\subsection{Special Cases}

Table 1 does not report any prediction of either account for cases 5 and 6 . The specificity of these two cases is that they denote situations wherein condition $m$ is satisfied whilst condition $p_{1}$ is not. These situations, however, cannot occur, and cases 5 and 6 cannot be constructed, because the satisfaction of condition $m$ entails that condition $p_{1}$ is satisfied, too.

Let us assume that condition $m$ is satisfied, i.e., that $<\neg x, \neg y>$ is ruled out by common knowledge as a model of 'If $x, y$.' That is, common knowledge dictates that $x$ and $y$ cannot be simultaneously false: one at least must be true. In such a case, the truth of $x$ is bound to have bearings on the epistemic relevance of asserting $y$, for this epistemic relevance is null when $x$ is false. This entails, by definition, the satisfaction of $p_{1}$.

Note that, in contrast, the satisfaction of $p_{1}$ does not entail the satisfaction of $m$. This was already illustrated by agreement case 3 and critical case 4 , in which $p_{1}$ was satisfied because the truth of $x$ had bearings on the practical (rather than epistemic) relevance of asserting $y$. It is not entirely clear, though, whether mental model theory has the same position on that issue, as one paragraph in JohnsonLaird and Byrne (2002) may suggest otherwise. In the course of discussing how the semantic contents of a conditional, as well as the context of its utterance, can block the construction of some possibilities, Johnson-Laird and Byrne (2002) define what they call the 'relevance' interpretation of a conditional: 
The relevance interpretation. Content or context precludes the possibility in the conditional interpretation in which the consequent does not occur. Typical examples occur when the antecedent asserts merely a condition to which the consequent may be relevant, for example, "If you are interested in Vertigo, then it is on TV tonight." Individuals know that the antecedent possibility has no bearing on the occurrence of the consequent, and so the consequent holds in any case. (page 662)

This definition may be read as suggesting that the possibility $<\neg x, \neg y>$ is discarded when $x$ is a condition to which $y$ may be relevant (or more precisely, we believe, the assertion of $y$ ). Thus, this definition may be interpreted as suggesting that $p_{1}$ entails $m$. What is not clear, under closer examination of the paragraph above, is why this should be the case. The crux of the definition is that individuals come to the assumption that $y$ holds in any case, based on some feature of the conditional: Either that 'the antecedent possibility has no bearing on the occurrence of the consequent' or that 'the antecedent asserts merely a condition to which the consequent may be relevant'. None of these two possibilities stands correct upon closer examination.

First, one cannot come to the conclusion that $y$ holds in any case (whether or not $x$ is the case) from the assertion that 'if $x, y$ ' and the belief that $x$ has no bearing on the occurrence of $y$. That would be a recipe for disaster:

(20) a. If we have pasta on Monday, the Mafia has weapons of mass destruction.

b. If it is cold on Tuesday, it is a good idea to put all our money in the 
stock market.

Clearly, we are not ready to conclude that the Mafia has weapons of mass destruction whether or not we have pasta on Monday, simply because we know that having pasta on Monday has no bearing on the Mafia having weapons of mass destruction.

Second, the fact that $x$ is a condition to which the assertion of $y$ is relevant cannot be taken as a guarantee that $y$ is true whether or not $x$. This point is treated in great detail by Siegel (2006), who offers in particular the following example:

If you want to hear a big fat lie, George W. and Condi Rice are secretly married.

Example (21) is a perfectly acceptable conditional, whose antecedent asserts merely a condition to which the assertion of its consequent is relevant, and whose antecedent has no bearing on the occurrence of the consequent. Nonetheless, it does not lead people to assume that George W. and Condi Rice are secretly married no matter what.

Note that a serious problem arises from the very idea that the context in which the conditional is uttered might block the possibility $<\neg x, \neg y>$. Quite uncontroversially, the context of a conditional includes what has been uttered just before the conditional itself was asserted. Let us suppose that $y$ is asserted just before 'if $x, y$ ' is asserted. Because $y$ is part of the context of the assertion that 'if $x, y$ ', context precludes that $\langle\neg x, \neg y\rangle$ is included in the interpretation of the conditional. As a consequence, 'if $x$ then $y$ ' does not throw away semantic information 
as compared to $y$. Therefore, on the own terms of mental model theory, allowing for the contextual blocking of $\langle\neg x, \neg y>$ implies that every instantiation of the paradoxical inference should become acceptable.

This being pointed out, whether or not the mental model characterisation of 'relevance' conditionals should be fixed or abandoned is not a question we can solve in this article. Our aim was rather to note that, under a superficial reading, mental model theory might be taken as suggesting that condition $p_{1}$ entailed condition $m$; but that there was no solid ground for that reading of the theory.

\section{Biscuits and Paradoxes}

In developing our dual pragmatic criterion, we have capitalised on the characteristics of 'biscuit' conditionals. In this section, we consider the possibility that all disappearances of the paradox may be imputable to the use of a biscuit conditional, and the related possibility that there does not exist any true exception to the paradox. ${ }^{8}$

We have argued that the absence of the paradox entails that $p_{1}$ is satisfied: The truth of $x$ has bearings on the relevance of asserting $y$. The question is, now, whether the satisfaction of $p_{1}$ entails that the conditional 'if $x, y$ ' is of the biscuit variety. Let us consider two diagnostic tests used to distinguish normal conditionals from biscuit conditionals (DeRose \& Grandy, 1999). The first consists of checking whether the question 'And what if $\neg x$ ?' makes sense upon hearing 'if $x, y$ ' ; the second test consists of checking whether the contraposition 'if $\neg y, \neg x$ '

\footnotetext{
${ }^{8}$ The arguments in this section owe greatly to one anonymous referee.
} 
makes any sense.

For all the conditionals in this article that satisfy $p_{1}$, the answer to both questions is 'No'. For example:
a. If you've not seen Amy yet, she's dyed her hair red.
b. ?And what if I have seen her?
c. ?If Amy has not dyed her hair red, you have seen her already.
(23) a. If Viv played a game, Viv did not play soccer.
b. ?And what if Viv did not play a game?
c. ?If Viv played soccer, she did not play a game.

Based on these diagnostics, it could be tempting to consider that all conditionals that satisfy $p_{1}$ qualify as biscuit conditionals. This conclusion, however, might be hasty. What is indisputable is that these conditionals do not qualify as normal conditionals; what is disputable is whether this makes them biscuit conditionals. While (22-a) and (23-a) both fail the question test and the contraposition test, they seem to do so for qualitatively different reasons.

More precisely, (23-a) fails both tests for semantic reasons. The fact that $\neg y$ is known to entail $x$ (soccer is a game) is responsible for the incongruity of asking whether $y$ would be true if $x$ was false, and also for the incongruity of asserting that $\neg y$ entails $\neg x$. No comparable semantic reasons can account for the fact that (22-a) fails both tests. It is an open question whether we should call (23-a) a biscuit conditional on the grounds that it fails the question test and the contraposition test, even though it does so for semantic reasons. If we make that choice, then 
the disappearance of the paradox (and thus the satisfaction of $p_{1}$ ) does entail the fact that the conditional is a biscuit; and the disappearance of the paradox can be added to the linguist's toolbox for diagnosing biscuit conditionals.

If really the disappearance of the paradox always involves a biscuit conditional, or a close cousin such as (23-a), then the possibility arises that there exists no true exception to the paradoxical character of ' $y$; so if $x, y$.' One agreed-upon characteristic of biscuit conditionals is that their consequents do not merely translate as the proposition they express. That is, the consequent $y$ in a biscuit if $x$, $y$ ' is a different semantic object from an independent occurrence of the same sentence ' $y$ ' (Siegel, 2006). Hence, the biscuit version of the argument ' $y$; so if $x$, $y$ ' might only have the superficial appearance of the paradox, but not its structure, for its structure would more appropriately be noted as ' $y_{1}$; so if $x, y_{2}$ '.

Thus, if all acceptable instances of the paradox involve biscuit, or biscuit-like conditionals; if specifically all these conditionals are such that their consequent is interpreted as a different semantic object than the independent occurrence of the same sentence; then the conclusion may be reached that there does not exist any true exception to the paradox, once the apparent exceptions are described at an appropriate level of semantic detail. This speculation, though, is left for future linguistic inquiry and not addressed further in this article, for it does not bear on our primarily psycholinguistic purposes. 


\section{Final Words}

We have offered a pragmatic account of when and why the inference from $y$ to 'if $x, y$ ' is paradoxical. We have shown that even though mental model theory (Johnson-Laird \& Byrne, 2002) explains the oddity of the paradox in terms of loss of semantic information, the way the explanation is operationalised in terms of mental models is inadequate, as the condition so formulated is neither necessary nor sufficient to explain the appearance and disappearance of the paradox.

In this article, we have focused on the mental model account as a contrast to our own pragmatic account. Mental model theory has a special burden of explaining the paradox, because the 'core meaning' it assigns to the natural language connective 'if' is akin to the material conditional. Theories that do not endorse that interpretation of 'if', however, do not necessarily endorse as valid the inference from $y$ to 'if $x, y$ ', and do not share the burden of explaining the paradox. Indeed, the paradoxes of material implication have always been, and remain, a part of the motivation for other accounts of conditionals, such as the Stalnaker-Lewis possible words semantics (Stalnaker, 1968), or psycholinguistic approaches (derived from philosophical work by, e.g., Adams, 1975; Edgington, 1995) such as the Bayesian account (Oaksford \& Chater, 2001, 2007) or the Suppositional account (Evans, 2007; Evans \& Over, 2004). These two major psycholingustic approaches allow a conditional 'If $x, y$ ' to be uncertain, assigning it the conditional probability $\operatorname{Pr}(y \mid x)$. Because the probability of the conditional 'If $x, y$ ' (the conclusion of the inference) may be smaller than that of $y$ (the premise of the inference), the inference from $y$ to 'If $x, y$ ' is not valid, which accounts for its intuitive oddity. 
However, a case of interest is that in which the premise $y$ is certain. In that case, 'If $x, y$ ' must also be certain, and the inference is valid. This specific case remained to be accounted for by probabilistic approaches to conditionals; they can now rely on our pragmatic account.

\section{References}

Adams, E. 1975: The logic of conditionals: An application of probability to deductive logic. Dordrecht: Reidel.

Austin, J. L. (1970) Ifs and cans. In Philosophical papers (2nd ed., pp. 205-32). Oxford: Clarendon Press.

Bonnefon, J. F., \& Villejoubert, G. (2007) Modus Tollens, Modus Shmollens: Contrapositive reasoning and the pragmatics of negation. Thinking and Reasoning, 13, 207-22.

DeRose, K., \& Grandy, R. E. (1999) Conditional assertions and "biscuit" conditionals. Noûs, 33, 405-20.

Edgington, D. (1995) On conditionals. Mind, 104, 235-329.

Evans, J. St. B. T. 2007: Hypothetical thinking: Dual processes in reasoning and judgment. Hove, England: Psychology Press.

Evans, J. St. B. T., \& Over, D. E. 2004: If. Oxford: Oxford University Press.

Johnson-Laird, P. N., \& Byrne, R. M. J. 1991: Deduction. Hillsdale, NJ: Lawrence Erlbaum Associates.

Johnson-Laird, P. N., \& Byrne, R. M. J. (2002) Conditionals: A theory of meaning, pragmatics, and inference. Psychological Review, 109, 646-78. 
Johnson-Laird, P. N., Byrne, R. M. J., \& Girotto, V. (2009) The mental model theory of conditionals: A reply to Guy Politzer. Topoi, 28, 75-80.

Oaksford, M., \& Chater, N. (2001) The probabilistic approach to human reasoning. Trends in Cognitive Sciences, 5, 349-57.

Oaksford, M., \& Chater, N. 2007: Bayesian rationality: The probabilistic approach to human reasoning. Oxford: Oxford University Press.

Politzer, G. (2007) Reasoning with conditionals. Topoi, 26, 79-95.

Politzer, G. (2009) Could it be the case that if I am right my opponents will be pleased? A rejoinder to Johnson-Laird, Byrne and Girotto. Topoi, 28, 81-5.

Siegel, M. E. A. (2006) Biscuit conditionals: Quantification over potential literal acts. Linguistics and Philosophy, 29, 167-203.

Sperber, D., \& Wilson, D. 1995: Relevance, communication and cognition. Oxford: Blackwell. (Original work published 1986)

Stalnaker, R. (1968) A theory of conditionals. In N. Rescher (Ed.), Studies in logical theory (pp. 98-112). Oxford: Blackwell. 